\title{
Delays in Post-Remission Chemotherapy for Philadelphia Chromosome Negative Acute Lymphoblastic Leukemia are Associated with Inferior Outcomes in Patients who Undergo Allogeneic Transplant: an Analysis from ECOG 2993/MRC UK ALLXII
}

\author{
Anita J. Kumar, MD MS ${ }^{1}$, Phyllis A. Gimotty, PhD², Joel Gelfand, MD MS ${ }^{3}$, Georgina Buck ${ }^{4}$, \\ Jacob M. Rowe, MD ${ }^{5,6}$, Anthony H Goldstone, MD $^{7}$, Adele Fielding, $\mathrm{PhD}^{8}$, David I Marks, \\ MD ${ }^{9}$, Mark Litzow, MD ${ }^{10}$, Elisabeth Paietta, PhD $^{11}$, Hillard M. Lazarus, MD ${ }^{12}$, Martin S. \\ Tallman, MD ${ }^{13}$, Selina M. Luger, MD ${ }^{14}$, and Alison W. Loren, MD MS ${ }^{15}$
}

${ }^{1}$ Division of Hematology/Oncology, Tufts University Medical Center, Boston, MA ${ }^{2}$ Center for Clinical Epidemiology and Biostatistics, Perelman School of Medicine at the University of Pennsylvania, Philadelphia, PA; pgimotty@mail.med.upenn.edu ${ }^{3}$ Department of Dermatology, University of Pennsylvania, Philadelphia, PA; joel.gelfand@uphs.upenn.edu ${ }^{4}$ Clinical Trial Service Unit, Oxford, United Kingdom; georgina.buck@ctsu.ox.ac.uk ${ }^{5}$ Rambam Medical Center, Haifa, Israel ${ }^{6}$ Eastern Cooperative Oncology Group, Brookline, MA; rowe@rambam.health.gov.il ${ }^{7}$ North London Cancer Network, Univ. College London Hosp., London, United Kingdom; anthony.goldstone@uclh.nhs.uk ${ }^{8}$ Haematology, University College London, London, United

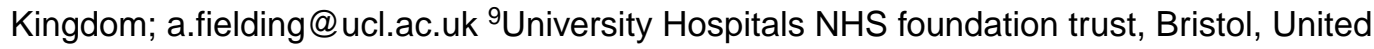
Kingdom; david.marks@ubht.swest.nhs.uk ${ }^{10}$ Mayo Clinic, Rochester, MN; litzow.mark@mayo.edu ${ }^{11}$ Cancer Center, The North Division of Montefiore Medical Center, Bronx, NY; epaietta@earthlink.net ${ }^{12}$ Case Comprehensive Cancer Center, Case Western Reserve University, Cleveland, OH; hml@case.edu ${ }^{13}$ Leukemia Service, Department of Medicine, Memorial SloanKettering Cancer Center, New York, NY; TallmanM@mskcc.org ${ }^{14}$ Abramson Cancer Center, University of Pennsylvania, Philadelphia, PA; Selina.luger@uphs.upenn.edu ${ }^{15}$ Abramson Cancer Center, University of Pennsylvania, Philadelphia, PA; Alison.loren@uphs.upenn.edu

\begin{abstract}
Adults with acute lymphoblastic leukemia (ALL) have a poorer prognosis than children due to a high risk of relapse. One explanation may be variable adherence to dose-intense chemotherapy. However, little is known about risk factors for delays in therapy and their impact on survival. We conducted an analysis of ECOG 2993/UKALLXII trial to study delays in post-remission chemotherapy in adults with newly-diagnosed ALL. Logistic regression was used to identify risk
\end{abstract}

Correspondence to: Phyllis A. Gimotty; Joel Gelfand; Georgina Buck.

Corresponding Author: Anita J. Kumar, MD, 800 Washington St, Box \#245, Boston, MA 02111, Phone 201-543-9115, akumar5@tuftsmedicalcenter.org. 
factors for a very long delay ( $>4$ weeks, VLD) in start of intensification therapy. Cox regression was used to evaluate the impact of delays on overall and event-free survival (OS, EFS).

We evaluated 1076 Philadelphia chromosome negative ( $\mathrm{Ph}-$ ) patients who completed induction chemotherapy, achieved complete remission, and started intensification. Factors independently associated with VLD included: duration of hospitalization (Odds Ratio $(\mathrm{OR})=1.2, \mathrm{p}<0.001$ ) during Phase I; thrombocytopenia during Phase I (OR=1.16, $\mathrm{p}=0.004)$ or Phase II (OR 1.13, $\mathrm{p}=0.001)$; chemotherapy dose reductions during induction Phase I (OR=1.72, $\mathrm{p}<0.014)$; female sex $(\mathrm{OR}=1.53, \mathrm{p}=0.010)$; Black $(\mathrm{OR}=3.24, \mathrm{p}=0.003)$ and Asian $(\mathrm{OR}=2.26, \mathrm{p}=0.021)$ race; and increasing age $(\mathrm{OR}=1.31, \mathrm{p}<0.001)$. In multivariate $\mathrm{Cox}$ regression, patients who underwent allogeneic stem cell transplant (alloHCT) had significantly worse OS (HR 1.4, p=0.03) and EFS (HR 1.4, $\mathrm{p}=0.02$ ) after experiencing a VLD compared to alloHCT patients who experienced $<=4$ weeks delay.

Specific populations (female, older, Black, and Asian patients) were more likely to experience delays in chemotherapy, as were those with significant toxicity during induction. Very long delays in therapy negatively affected outcomes in patients undergoing allografting.

\section{Keywords}

acute lymphoblastic leukemia; chemotherapy; allogeneic stem cell transplant

\section{INTRODUCTION}

Acute lymphoblastic leukemia (ALL) is an aggressive hematologic malignancy treated with dose-intense chemotherapy with or without allogeneic hematopoietic stem cell transplant (HCT). Despite advances in chemotherapy and supportive care, pediatric patients experience better long-term outcomes than adult patients. While $90 \%$ of pediatric patients experience 5year event-free survival (EFS), only $30-50 \%$ of adults achieve this milestone.(1,2) These differences are partially attributable to a higher proportion of adults with biologically highrisk disease, particularly poor risk cytogenetics such as the Philadelphia ( $\mathrm{Ph})$ chromosome.

Variations in administration of therapy between pediatric and adult regimens may also account for poorer outcomes among adult ALL patients. Adolescent and young adult patients (AYAs), who may be treated by either adult or pediatric oncologists, have been studied to better understand the contribution of treatment differences to inferior outcomes for adults with ALL. Retrospective studies identify key differences that may contribute to improved overall survival (OS) for AYAs on pediatric protocols that include 1) AYAs receive higher doses of chemotherapy, especially non-myelosuppressive drugs and 2) have shorter intervals between treatment courses.(6-11) Hence, treatment differences, including delays in chemotherapy administration, may result in poorer outcomes for adult patients.(12)

While the need for dose-intense chemotherapy in curing hematologic malignancies is well described, there is scant literature describing risk factors for and outcomes after chemotherapy delays.(13-15) There is a presumption that delaying chemotherapy due to toxicity is safer for patients in the short-term, but there are few data that investigate long- 
term consequences such as disease-free and OS. $(16,17)$ In this study, we investigate risk factors for delay in intensification start for adults who underwent therapy on ECOG2993/ UKALLXII, the largest prospective trial for newly diagnosed adult ALL. We also examine whether delay is associated with poorer OS and EFS.

\section{METHODS}

We performed an analysis of ECOG 2993/MRC UKALLXII, an international clinical trial that enrolled 2109 newly diagnosed adult ALL patients age 20-65 between 1993-2006.(18) Patients received a 2-phase induction from Day 1-56, followed by a mandatory rest period (14 days for MRC, 28 days for ECOG) prior to starting intensification therapy with highdose methotrexate (Supp. Figure 1). This trial was registered at http://www.clinicaltrials.gov as NCT00002514. Ph- patients who achieved a complete remission (CR) after induction and had a documented intensification start date were included in the current analysis. Patients who were not in $\mathrm{CR}$, died, or went off protocol prior to intensification were excluded, as were those with incomplete documentation for dates of chemotherapy.

The primary outcome was the duration of delay in post-remission intensification therapy. The following potential reasons for delay were evaluable in the database: duration of neutropenia, thrombocytopenia, hospitalization; patient age; race, cranial-spinal irradiation for CNS disease, chemotherapy dose reductions, infection, and time to CR. The secondary outcome was to evaluate whether delay in intensification start was associated with decreased OS and EFS. OS was defined as time from intensification start to death from any cause. EFS was defined as time from intensification start to relapse or death from any cause. Patients who were alive (and for EFS, in remission) were censored at date last seen. We chose to use intensification start as the start of survival analysis to 1) satisfy the proportional hazard ratio assumption and;2) avoid potential bias when using the date of diagnosis as the survival start point, since the most-delayed patients would have increased survival due to longer delay.

Per protocol, patients started intensification on Day+71 (MRC) or Day+85 (ECOG), so delay was initially defined as number of days beyond 71 (MRC) or 85 (ECOG) days to intensification start. With this definition, however, 1071/1076 (99.5\%) patients were delayed, with a median time to intensification start of 100 days (range 72-477). Therefore, patients were categorized into three delay groups: short delay (SD, $<=98$ days from start of induction to intensification), intermediate delay (ID, 99-112 days) and very long delay (VLD, >112 days from start of induction to intensification) for both MRC and ECOG patients, allowing both groups 56 days induction and 28 days rest (total 84 days) prior to entering a period of delay. Patients with long delay (LD) were those patients with either ID or VLD. Hence, LD and VLD corresponded with at least a 2 or 4-week delay beyond 84 days of induction and rest per protocol for both MRC and ECOG cohorts.

Univariate logistic regression was used to compare LD to SD, and VLD to SD + ID, based on demographic factors (age, sex, race) and induction complications (infection, dose reductions, $\mathrm{CR}$ in $>28$ days, $\mathrm{CNS}$ disease requiring cranial-spinal radiation). The univariate analysis was used to identify predictors significantly associated with VLD to include in the multivariate analysis. A p-value of $\leq 0.05$ in the univariate analysis was considered 
statistically significant. Multivariate logistic regression was then used to identify predictors of VLD. This was done using backward elimination: by removing the weakest nonsignificant predictor until all predictors that appear in the reduced model were significant. The same variables were included in the multivariate logistic regression model for LD. We also explored interactions between the main effect and other significant explanatory variables, such as age and sex. Approximately $10 \%$ of the patients (105/1076) had some missing data for at least one of the continuous variables of interest, and a sensitivity analysis using mean imputation was run to assess the effect of missing variables. (19)

Survival curves for OS and EFS were estimated using the Kaplan-Meier method, and survival curves for delay groups were compared with the log-rank test. Cox proportional hazards regression models were used to estimate OS and EFS hazard ratios (HR) and 95\% confidence intervals (CI) within subgroups defined by post-remission therapy (allogeneic HCT in CR1, autologous SCT, non-transplant therapy). Schoenfeld residuals were used to test for the proportional hazard assumption, and there were no violations of the assumption. Multivariate Cox regression models were used to adjust for known high-risk disease features associated with poorer survival: older age, high-risk WBC at diagnosis, and lineage. Interactions between the main effect and post-remission treatment were investigated. Since patients allocated to the autologous HCT arm of the study demonstrated inferior outcomes in an interim analysis, this arm was excluded from the OS/EFS analysis, but was evaluated for the endpoint of risk factors for VLD/LD. Analysis was performed with STATA 12.1 (STATA Corporation, College Station, TX).

Institutional review board approval exemption was obtained prior to conducting this study. This manuscript was prepared according to the Strengthening the Reporting of Observational Studies in Epidemiology recommendations.(20)

\section{RESULTS}

\section{Patient Characteristics}

There were 1076 patients in our analysis (Supp. Figure 2). Demographics and clinical characteristics are outlined in Table I. Missing data rates were $<2 \%$ for all variables except for data reported for evaluation for central nervous system (CNS) disease requiring cranialspinal radiation. This information was not documented in 290 cases (27\%). Median age was 28 years (Interquartile range, IQR 20-40). Over $80 \%$ of patients achieved CR by day 28 of induction.

\section{Risk Factors for VLD}

Of the 1076 patients included in this analysis, 270 (25\%) had VLD and 577 (54\%) had LD. In a univariate analysis, female patients $(\mathrm{OR}=1.72 ; \mathrm{p}<0.001)$, Black patients $(\mathrm{OR}=2.70$; $\mathrm{p}=0.013$ ), and older patients (evaluated by decade, $\mathrm{OR}=1.39 ; \mathrm{p}<0.001$ ) were each associated with increased risk of experiencing VLD. During induction Phase I, dose reductions and infection were associated with VLD $(\mathrm{OR}=1.83 ; \mathrm{p}=0.002$ and $\mathrm{OR}=1.80 ; \mathrm{p}<0.001$, respectively). However, during induction Phase II these variables were not significant predictors of VLD (dose reduction Phase II OR=1.01; $\mathrm{p}=0.94$; Infection during induction 
Phase II OR 1.14; $\mathrm{p}=0.35$ ). In addition, during Phase I and Phase II of induction, duration (in weeks) of hospitalization (Phase I OR=1.29; $\mathrm{p}<0.001$; Phase II OR=1.10; $\mathrm{p}=0.001$ ), neutropenia (Phase I OR=1.15; $\mathrm{p}=0.002$; Phase II OR=1.17; $\mathrm{p}<0.001$ ), and thrombocytopenia (Phase I OR=1.27, $\mathrm{p}<0.001$ and Phase II OR=1.19; $\mathrm{p}<0.001$ ) were associated with VLD. Failure to achieve a CR at 4 weeks and cranial-spinal irradiation for CNS disease were not significantly associated with VLD (OR=1.01; $\mathrm{p}=0.98$ and $\mathrm{OR}=0.97$; $\mathrm{p}=0.82$, respectively). There were no statistically significant interactions for VLD. (Table II)

In the reduced multivariate logistic regression for VLD (Table II, $n=1041$ ), three patient characteristics were associated with VLD: 1) Sex: women were more likely to have VLD compared to men (rates $32 \%$ versus $21 \%$, OR 1.53, CI 1.11-2.1, p=0.01); 2) Race: Black patients (rates $85 \%$ versus $52 \%$, OR 3.24, CI 1.41-7.45, $\mathrm{p}=0.003$ ) and Asian patients (rates $64 \%$ versus $52 \%$, OR 2.26 , CI 1.13-4.52, $\mathrm{p}=0.021$ ) were more likely to have VLD compared to white patients; and 3 ) increasing age (decades) was associated with VLD (OR 1.31, CI $1.16-1.49, \mathrm{p}<0.001)$. The other significant predictors in the model were: weeks in the hospital Phase I (OR 1.2, CI 1.11-1.31, p<0.001), weeks of thrombocytopenia (Phase I OR 1.16, CI 1.05-1.28, p=0.004; Phase II OR 1.13, CI 1.05-1.22, p=0.001), and chemotherapy dose reductions during Phase I (OR 1.72, CI 1.12-2.64, $\mathrm{p}=0.014$ ). Multivariate logistic regression for LD yielded similar results, except that Asian race and Phase II duration of thrombocytopenia were not statistically significant (Table II). A sensitivity analysis was done to determine the effect of missing data on multivariate regression and the results were similar to the original multivariate with no new predictors of LD or VLD.

\section{Overall Survival}

Of the 909 patients included in survival analysis (alloHCT $=419$, non-transplant=490), 465 $(51.1 \%)$ patients died. Median OS from intensification start was 42.6 months (IQR 12.1-95.5). As delay increased, there was a corresponding decrease in OS ( $\mathrm{p}=0.011$, Figure 1A). The same relationship was seen in both alloHCT and non-transplant settings (alloHCT $\mathrm{HR}=1.19 ; \mathrm{p}=0.045$; non-transplant $\mathrm{HR}=1.19, \mathrm{p}=0.021$ ). Using Cox regression, the HR associated with VLD compared to ID and SD was significant in both alloHCT and nontransplant arms (alloHCT HR=1.47, p=0.024; non-transplant $\mathrm{HR}=1.41, \mathrm{p}=0.024$ ), whereas there was no significant difference in HR between ID and SD (alloHCT HR=1.03, $\mathrm{p}=0.86$; non-transplant $\mathrm{HR}=1.31, \mathrm{p}=0.092$ ), so ID and $\mathrm{SD}$ were combined into one cohort that was delayed $<=4$ weeks.

In alloHCT patients, VLD was associated with poorer median OS compared to delay $<=4$ weeks (SD+ID), (25.4 versus 47.6 months, $\mathrm{p}=0.018$, Figure 1B). In non-transplant patients, a VLD was associated with a non-significant reduction in median OS (38.3 versus 43.2 months, $\mathrm{p}=0.08$, Figure 1C). VLD was not associated with longer median time from intensification start to alloHCT ( 76 vs. 74 days, $p=0.67$ ). Of the 220/419 patients who died after alloHCT, VLD (50/220) was associated with shorter time to death after alloHCT (logrank $\mathrm{p}=0.044$, Figure 1D). Cause of death was not significantly different between VLD and non-VLD patients (relapse in $27 \%$ of VLD versus $31 \%$ of non-VLD patients; treatmentrelated mortality in $73 \%$ of VLD versus $69 \%$ of non-VLD patients, $\mathrm{p}=0.55$ ). 
In a multivariate Cox regression that included other high-risk features for decreased OS for adults with ALL (B-lineage, elevated WBC, age), for patients who underwent alloHCT, VLD remained significantly associated with poorer OS $(\mathrm{HR}=1.43$; $\mathrm{CI}=1.04-1.97, \mathrm{p}=0.027)$. For non-transplant patients, the adjusted HR for VLD was not statistically significant $(\mathrm{HR}=1.12, \mathrm{CI}=0.85-1.28, \mathrm{p}=0.41)$ (Table III).

\section{Event Free Survival}

Fifty-five percent of patients (500/909) died or relapsed. Median EFS was 36.7 months (IQR 7.5-90.5). Seven patients had documented relapse prior to intensification start but proceeded with intensification (alloHCT=4, non-transplant=3). As delay increased, there was a corresponding decrease in EFS ( $\mathrm{p}=0.005$, Supp. Figure $3 \mathrm{~A}$ ). The same relationship was seen in both alloHCT and non-transplant patients (alloHCT HR=1.19, $\mathrm{p}=0.038$; non-transplant $\mathrm{HR}=1.22, \mathrm{p}=0.007$ ). After a VLD, alloHCT patients had poorer EFS than those delayed $<=4$ weeks (median 12.8 vs. 39.5 months, $\mathrm{p}=0.024$, Supp. Figure 3B). Patients who received nontransplant post remission therapy had poorer EFS after VLD, which was marginally statistically significant (median 32.4 months vs. 41.0 months, p=0.05, Supp. Figure 3C).

In a multivariate Cox regression, adjusted for age by decade, lineage, and high-risk WBC at diagnosis, for alloHCT patients VLD was associated with poorer EFS (HR 1.43; CI 1.05-1.95, $\mathrm{p}=0.024)$. For non-transplant patients, the effect of VLD was not statistically significant: $\mathrm{p}=1.16$ (CI 0.88-1.52, $\mathrm{p}=0.28$ ) (Table III).

\section{DISCUSSION}

In the largest prospective trial of adults with ALL, we were able to identify several risk factors for chemotherapy delays and to show that these delays are linked to inferior outcomes. Specifically, delays in initiation of post-remission intensification occur nearly universally and more frequently occur in older, female, Black and Asian patients as well as those who experience excess toxicity from initial chemotherapy. For each decade of increasing age there was a nearly $40 \%$ increase in odds of VLD, suggesting that poorer outcomes experienced with increasing age may be due in part to greater incidence of longer delays. Although prolonged thrombocytopenia during induction was associated with longer delays, neutropenia was not. The trial allowed G-CSF, which may have facilitated marrow recovery to the required WBC of 3,000/uL to start intensification. Hence, G-CSF may be helpful in preventing delays even if it does not reduce infection rate or mortality.(21) (22) While it is unreasonable to suggest that physicians should continue on-schedule therapy for patients with active therapy complications such as sepsis or organ failure, our findings suggest a thoughtful re-evaluation of the feasibility of induction via two continuous phases in the adult population, particularly in older patients, in comparison with AYA protocols.

Black patients had a significantly higher risk of experiencing LD and VLD, and Asians had higher risk of VLD. Retrospective and population studies have demonstrated poorer survival rates among African-American cancer patients compared to Caucasian patients, despite improved 5-year cancer survival rates in all patients.(23) (24) (25) It has been suggested that poorer survival rates may not extend to minority patients enrolled in clinical trials, potentially reflecting biases in patients who enroll and suggesting that support services 
offered in a clinical trial setting that may improve therapy adherence.(26) Adult studies do not measure whether suboptimal adherence to dose-intense therapy is associated with survival outcomes, whereas the pediatric literature demonstrates that poorer adherence to maintenance oral 6-mercaptopurine therapy is seen in African-American and Asian pediatric patients, and may be associated with relapse.(27) In pediatric ALL there are clear survival differences in minority populations, which may be due to genetic predispositions, environmental factors, and/or access to care. (28-30) Our study shows that adult minority patients also face risks for delays in dose-intense therapy and these risks may partially explain poorer survival outcomes observed in population studies. Further analyses are needed to better understand these risk factors in adult minorities with ALL.

While there is population and clinical trial evidence that males have poorer survival for leukemia, there is little data on disparities in care by sex for adults with leukemia. $(18,31)$ We find that, even after adjusting for older age, females experienced more frequent delays. The reason for this is unknown - one could postulate that $\mathrm{LD}$ in females may be related to childcare planning or family support. It is not known whether patients were married or in a marriage-like relationship, which may impact social support at home and influence delay.

Our study confirms that longer delays are associated with decreased OS and EFS. This effect was particularly robust in the alloHCT population: those who experienced VLD were $40 \%$ more likely to relapse or die even after adjusting for older age, lineage, and high-risk WBC. It is not clear why a $>4$-week delay (rather than 2-4 weeks) was the critical duration to negatively impact survival. VLD may be a quantitative marker for poor patient performance status or sensitivity to chemotherapy toxicity. Pharmacokinetic and pharmacogenomic studies (with the exception of thiopurine methyltransferase testing in patients with myelosuppression on mercaptopurine) are typically not performed in adult ALL patients. Unplanned treatment delays may represent a biologic marker of intolerance to cytotoxic agents, predicting a worse outcome in high-risk procedures such as alloHCT. While there are several risk assessment models for mortality with alloHCT based on medical co-morbidities and disease status, delays during induction may also identify patients who are particularly intolerant to cytotoxic chemotherapy and may have additional uncaptured risk with alloHCT. (33) (34)

Delay could have been a factor in choosing whether to pursue alloHCT, as the registration to the alloHCT arm occurred just prior to intensification start. Physicians may have opted for alloHCT for delayed patients who were not originally assigned to alloHCT arm, feeling that they did not receive optimal dose-intense therapy. The effect of VLD could not be overcome despite intensified therapy for these patients. It is important to note that delays in intensification start should not be attributable to transplant planning logistics, as the registration to the alloHCT arm was made after the delay period, so transplant logistics (referral to transplant center, insurance approval) would have occurred after our measured delay.

There was a suggestion that VLD may have negatively impacted survival in patients who received non-transplant therapy. In particular, after 4 years, there is divergence in survival 
between VLD and non-VLD patients. Such a late divergence may reflect that delayed patients are dying from other causes, or that they may be a sicker population in general.

Although not part of our intended analysis, we also observed significant deviations from planned therapy after intensification. Of the 490 non-transplant patients, only 324/490 (66\%) were documented to start consolidation. 262 of these 324 patients $(81 \%)$ completed consolidation. 196 patients started maintenance therapy, and only 65/490 non-transplant therapy patients $(26 \%)$ are recorded to have completed maintenance. This suggests that delays and non-adherence likely extend to continued chemotherapy after remission and that the non-transplant patients may not have received optimal therapy on an ongoing basis.

The ECOG2993/UKALLXII trial did not document many details about induction toxicity and chemotherapy delays. Data that was not collected include reasons for prolonged hospitalization and chemotherapy dose reductions. Interestingly, although intensification was clearly delayed based on dates, many of these patients were not identified as "delayed," which is why reasons for delays were also frequently not noted. The trial did not evaluate ongoing performance status, co-morbidities, and medical events experienced prior to intensification start. We have no information on marital status and financial/employment information, insurance status, or other sociodemographic features. Disparities by sex and race in our study suggest that social risk factors likely exist. Our survival analysis did not incorporate high-risk cytogenetics due to significant missing data. A sub-analysis with data on 419 alloHCT patients did demonstrate, however, that VLD was still associated with poorer OS and EFS after accounting for MLL rearrangements. Lastly, as our analysis was restricted to patients on the ECOG 2993/UKALLXII protocol, it is difficult to quantify risks of delays in other ALL treatment regimens, including those administered off protocol.

In conclusion, we observe that in adults with ALL, specific patient populations (older, female, Black, Asian, and those experiencing significant chemotherapy toxicity) are at risk for experiencing lengthy delays in initiation of post-remission treatment. In an adjusted analysis, VLD is associated with poorer OS and EFS; this effect is most pronounced in patients who underwent alloHCT. Further studies are needed to identify potentially modifiable risk factors, so that interventions may be offered to optimize adherence to these intensive chemotherapy regimens, which we expect will contribute to more favorable longterm outcomes for adults with ALL.

\section{Supplementary Material}

Refer to Web version on PubMed Central for supplementary material.

\section{REFERENCES}

1. Pui CH, Evans WE. Treatment of acute lymphoblastic leukemia. The New England journal of medicine. 2006; 354:166-178. [PubMed: 16407512]

2. Rijneveld AW, van der Holt B, Daenen SM, Biemond BJ, de Weerdt O, Muus P, Maertens J, Mattijssen V, Demuynck H, Legdeur MC, Wijermans PW, Wittebol S, Spoelstra FM, Dekker AW, Ossenkoppele GJ, Willemze R, Cornelissen JJ. Intensified chemotherapy inspired by a pediatric regimen combined with allogeneic transplantation in adult patients with acute lymphoblastic leukemia up to the age of 40. Leukemia. 2011; 25:1697-1703. [PubMed: 21647160] 
3. Boucheix C, David B, Sebban C, Racadot E, Bene MC, Bernard A, Campos L, Jouault H, Sigaux F, Lepage E, et al. Immunophenotype of adult acute lymphoblastic leukemia, clinical parameters, and outcome: an analysis of a prospective trial including 562 tested patients (LALA87). French Group on Therapy for Adult Acute Lymphoblastic Leukemia. Blood. 1994; 84:1603-1612. [PubMed: 8068949]

4. Pui CH, Relling MV, Downing JR. Acute lymphoblastic leukemia. The New England journal of medicine. 2004; 350:1535-1548. [PubMed: 15071128]

5. Thomas X, Le QH. Prognostic factors in adult acute lymphoblastic leukemia. Hematology. 2003; 8:233-242. [PubMed: 12911941]

6. Ramanujachar R, Richards S, Hann I, Webb D. Adolescents with acute lymphoblastic leukaemia: emerging from the shadow of paediatric and adult treatment protocols. Pediatric blood \& cancer. 2006; 47:748-756. [PubMed: 16470520]

7. Woods WG, Franklin AR, Alonzo TA, Gerbing RB, Donohue KA, Othus M, Horan J, Appelbaum FR, Estey EH, Bloomfield CD, Larson RA. Outcome of adolescents and young adults with acute myeloid leukemia treated on COG trials compared to CALGB and SWOG trials. Cancer. 2013; 119:4170-4179. [PubMed: 24104597]

8. Stock W, La M, Sanford B, Bloomfield CD, Vardiman JW, Gaynon P, Larson RA, Nachman J. What determines the outcomes for adolescents and young adults with acute lymphoblastic leukemia treated on cooperative group protocols? A comparison of Children's Cancer Group and Cancer and Leukemia Group B studies. Blood. 2008; 112:1646-1654. [PubMed: 18502832]

9. de Bont JM, Holt B, Dekker AW, van der Does-van den Berg A, Sonneveld P, Pieters R. Significant difference in outcome for adolescents with acute lymphoblastic leukemia treated on pediatric vs adult protocols in the Netherlands. Leukemia. 2004; 18:2032-2035. [PubMed: 15483674]

10. Hallbook H, Gustafsson G, Smedmyr B, Soderhall S, Heyman M. Treatment outcome in young adults and children $>10$ years of age with acute lymphoblastic leukemia in Sweden: a comparison between a pediatric protocol and an adult protocol. Cancer. 2006; 107:1551-1561. [PubMed: 16955505]

11. Ramanujachar R, Richards S, Hann I, Goldstone A, Mitchell C, Vora A, Rowe J, Webb D. Adolescents with acute lymphoblastic leukaemia: outcome on UK national paediatric (ALL97) and adult (UKALLXII/E2993) trials. Pediatric blood \& cancer. 2007; 48:254-261. [PubMed: 16421910]

12. Schiffer CA. Differences in outcome in adolescents with acute lymphoblastic leukemia: a consequence of better regimens? Better doctors? Both? Journal of clinical oncology : official journal of the American Society of Clinical Oncology. 2003; 21:760-761. [PubMed: 12610169]

13. Wood WC, Budman DR, Korzun AH, Cooper MR, Younger J, Hart RD, Moore A, Ellerton JA, Norton L, Ferree CR, et al. Dose and dose intensity of adjuvant chemotherapy for stage II, nodepositive breast carcinoma. The New England journal of medicine. 1994; 330:1253-1259. [PubMed: 8080512]

14. Mayer RJ, Davis RB, Schiffer CA, Berg DT, Powell BL, Schulman P, Omura GA, Moore JO, McIntyre OR, Frei E 3rd. Intensive postremission chemotherapy in adults with acute myeloid leukemia. Cancer and Leukemia Group B. The New England journal of medicine. 1994; 331:896903.

15. Bonadonna G, Valagussa P. Dose-response effect of adjuvant chemotherapy in breast cancer. The New England journal of medicine. 1981; 304:10-15. [PubMed: 7432433]

16. Nagel CI, Backes FJ, Hade EM, Cohn DE, Eisenhauer EL, O'Malley DM, Fowler JM, Copeland LJ, Salani R. Effect of chemotherapy delays and dose reductions on progression free and overall survival in the treatment of epithelial ovarian cancer. Gynecologic oncology. 2012; 124:221-224. [PubMed: 22055764]

17. Bayraktar UD, Chen E, Bayraktar S, Sands LR, Marchetti F, Montero AJ, Rocha-Lima CM. Does delay of adjuvant chemotherapy impact survival in patients with resected stage II and III colon adenocarcinoma? Cancer. 2011; 117:2364-2370. [PubMed: 24048783]

18. Rowe JM, Buck G, Burnett AK, Chopra R, Wiernik PH, Richards SM, Lazarus HM, Franklin IM, Litzow MR, Ciobanu N, Prentice HG, Durrant J, Tallman MS, Goldstone AH. Induction therapy for adults with acute lymphoblastic leukemia: results of more than 1500 patients from the 
international ALL trial: MRC UKALL XII/ECOG E2993. Blood. 2005; 106:3760-3767. [PubMed: 16105981]

19. Groenwold RH, Donders AR, Roes KC, Harrell FE Jr. Moons KG. Dealing with missing outcome data in randomized trials and observational studies. Am J Epidemiol. 2012; 175:210-217. [PubMed: 22262640]

20. von Elm E, Altman DG, Egger M, Pocock SJ, Gotzsche PC, Vandenbroucke JP, Initiative S. The Strengthening the Reporting of Observational Studies in Epidemiology (STROBE) statement: guidelines for reporting observational studies. Lancet. 2007; 370:1453-1457. [PubMed: 18064739]

21. Lehrnbecher T, Zimmermann M, Reinhardt D, Dworzak M, Stary J, Creutzig U. Prophylactic human granulocyte colony-stimulating factor after induction therapy in pediatric acute myeloid leukemia. Blood. 2007; 109:936-943. [PubMed: 17008536]

22. Hornedo J, Sola C, Solano C, Lopez JJ, Alonso S, Lluch A, Ojeda B, Garcia-Conde J, CortesFunes $\mathrm{H}$. The role of granulocyte colony-stimulating factor (G-CSF) in the post-transplant period. Bone marrow transplantation. 2002; 29:737-743. [PubMed: 12040470]

23. Byrne MM, Halman LJ, Koniaris LG, Cassileth PA, Rosenblatt JD, Cheung MC. Effects of poverty and race on outcomes in acute myeloid leukemia. American journal of clinical oncology. 2011; 34:297-304. [PubMed: 20562587]

24. Patel MI, Ma Y, Mitchell BS, Rhoads KF. Understanding disparities in leukemia: a national study. Cancer causes \& control : CCC. 2012; 23:1831-1837. [PubMed: 22971999]

25. Pulte D, Redaniel MT, Jansen L, Brenner H, Jeffreys M. Recent trends in survival of adult patients with acute leukemia: overall improvements, but persistent and partly increasing disparity in survival of patients from minority groups. Haematologica. 2013; 98:222-229. [PubMed: 22929974]

26. Albain KS, Unger JM, Crowley JJ, Coltman CA Jr. Hershman DL. Racial disparities in cancer survival among randomized clinical trials patients of the Southwest Oncology Group. Journal of the National Cancer Institute. 2009; 101:984-992. [PubMed: 19584328]

27. Bhatia S, Landier W, Hageman L, Kim H, Chen Y, Crews KR, Evans WE, Bostrom B, Casillas J, Dickens DS, Maloney KW, Neglia JP, Ravindranath Y, Ritchey AK, Wong FL, Relling MV. Adherence to oral 6-mercaptopurine in African American and Asian children with acute lymphoblastic leukemia: a Children's Oncology Group study. Blood. 2014

28. Lim JY, Bhatia S, Robison LL, Yang JJ. Genomics of racial and ethnic disparities in childhood acute lymphoblastic leukemia. Cancer. 2014; 120:955-962. [PubMed: 24382716]

29. Goggins WB, Lo FF. Racial and ethnic disparities in survival of US children with acute lymphoblastic leukemia: evidence from the SEER database 1988-2008. Cancer causes \& control : CCC. 2012; 23:737-743. [PubMed: 22450738]

30. de Smith AJ, Walsh KM, Ladner MB, Zhang S, Xiao C, Cohen F, Moore TB, Chokkalingam AP, Metayer C, Buffler PA, Trachtenberg EA, Wiemels JL. The role of KIR genes and their cognate HLA class I ligands in childhood acute lymphoblastic leukemia. Blood. 2014; 123:2497-2503. [PubMed: 24518758]

31. Cook MB, McGlynn KA, Devesa SS, Freedman ND, Anderson WF. Sex disparities in cancer mortality and survival. Cancer epidemiology, biomarkers \& prevention : a publication of the American Association for Cancer Research, cosponsored by the American Society of Preventive Oncology. 2011; 20:1629-1637.

32. Scott, Huntington; Shah, NE.; Andrew; Loren, AW. The Impact of Marital and Socioeconomic Status on Outcomes of Patients with Acute Promyelocytic Leukemia. Blood. 2014; 124:12821282.

33. Armand P, Gibson CJ, Cutler C, Ho VT, Koreth J, Alyea EP, Ritz J, Sorror ML, Lee SJ, Deeg HJ, Storer BE, Appelbaum FR, Antin JH, Soiffer RJ, Kim HT. A disease risk index for patients undergoing allogeneic stem cell transplantation. Blood. 2012; 120:905-913. [PubMed: 22709687]

34. Sorror ML, Maris MB, Storb R, Baron F, Sandmaier BM, Maloney DG, Storer B. Hematopoietic cell transplantation (HCT)-specific comorbidity index: a new tool for risk assessment before allogeneic HCT. Blood. 2005; 106:2912-2919. [PubMed: 15994282] 

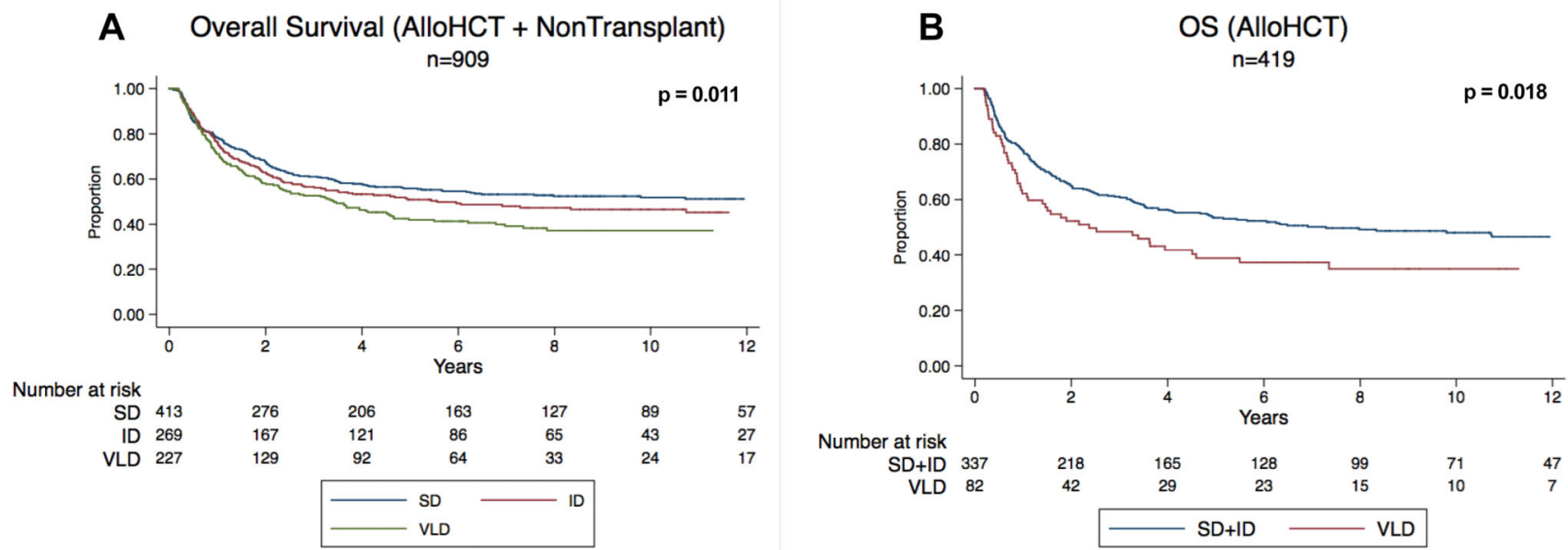

C

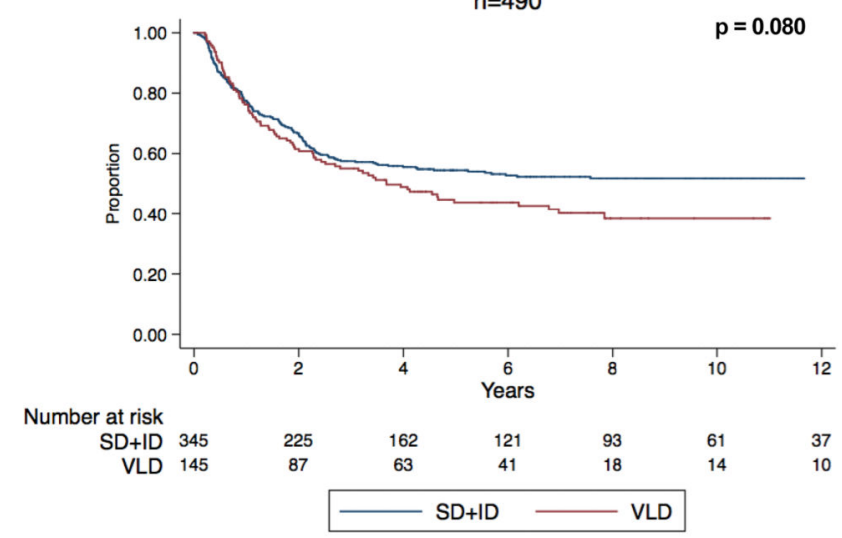

OS (NonTransplant)

$\mathrm{n}=490$
D

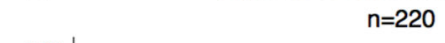

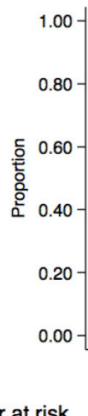

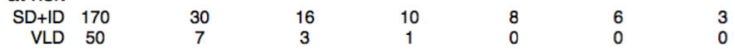

Figure 1.

OS: overall survival; VLD: very long delay; SD: short delay; LD: long delay 


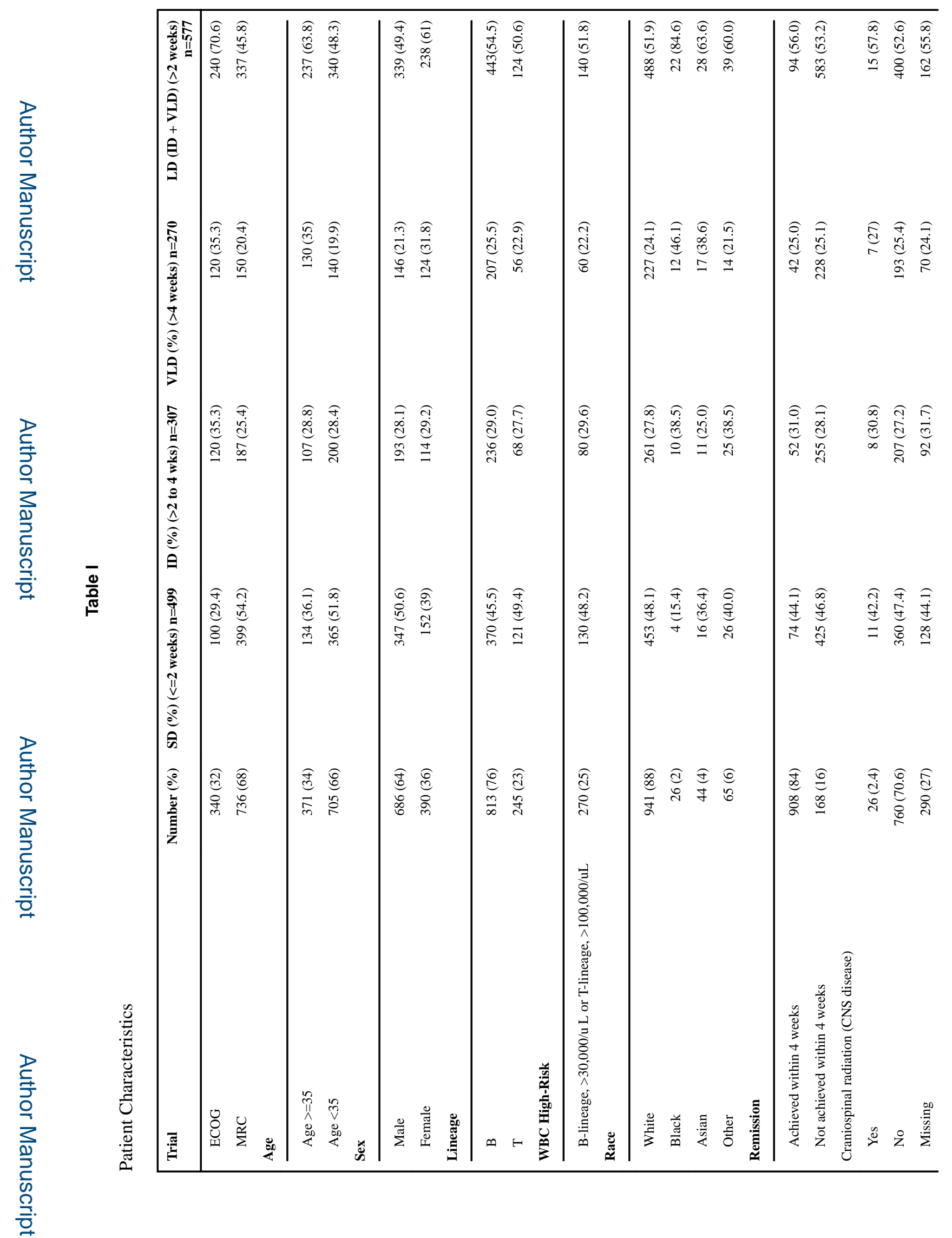

Am J Hematol. Author manuscript; available in PMC 2016 November 01. 


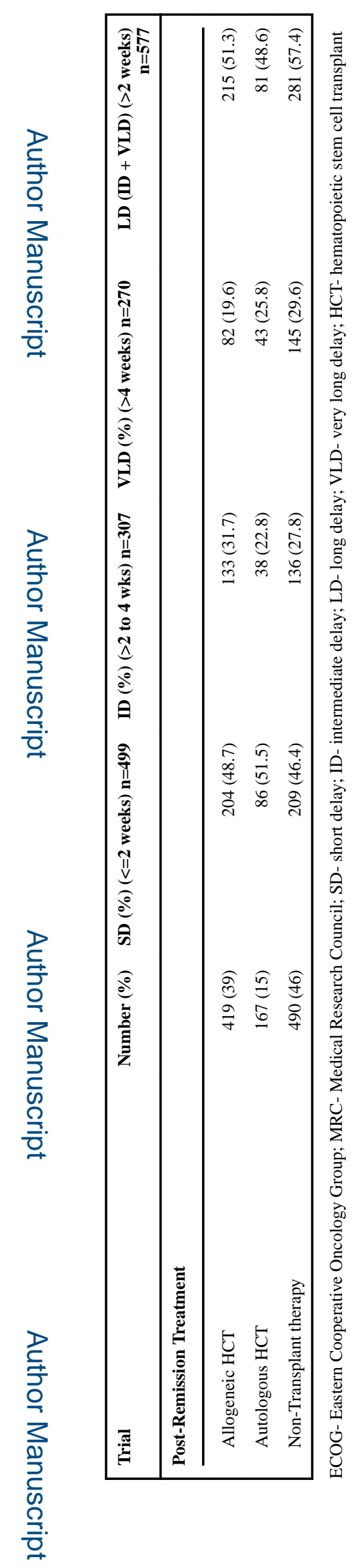




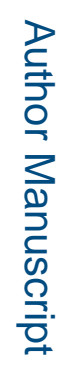

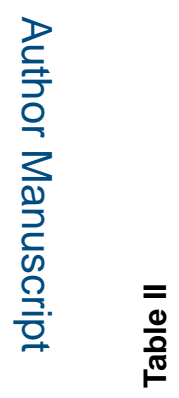

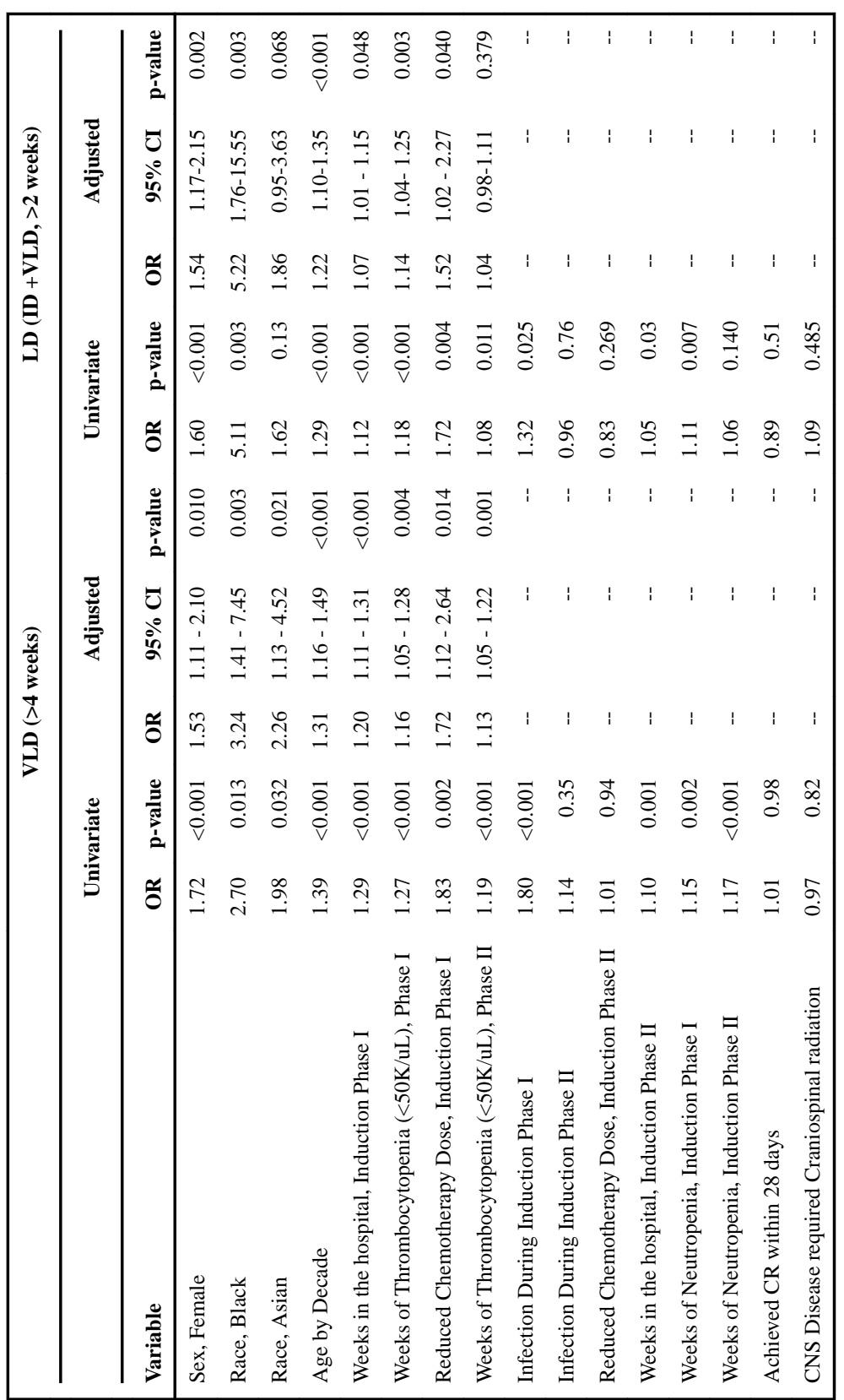

Am J Hematol. Author manuscript; available in PMC 2016 November 01. 


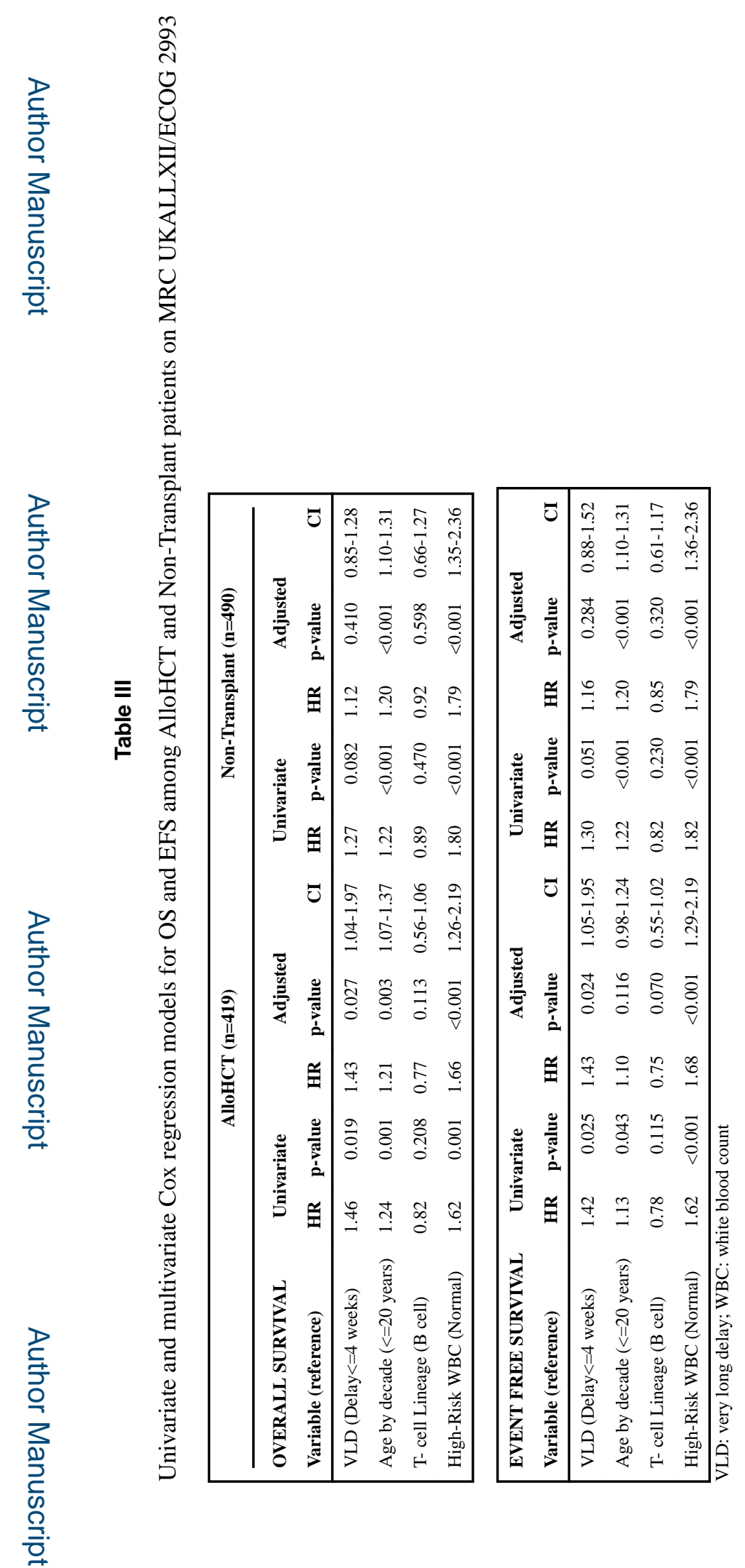

Am J Hematol. Author manuscript; available in PMC 2016 November 01. 